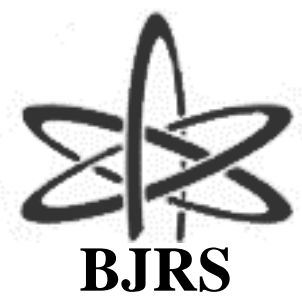

BRAZILIAN JOURNAL

$\mathrm{OF}$

RADIATION SCIENCES

08-03B (2021) 01-12

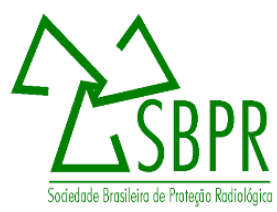

\title{
A Continuous Energy Neutron Transport Monte Carlo Simulator Project: Decomposition of the Neutron Energy Spectrum by Target Nuclei Tagging
}

\author{
L. F. Chaves Barcellos ${ }^{\text {a }}$ B. E. Bodmann ${ }^{\text {a }}$ S. Q. Bogado Leite ${ }^{\text {b }}$ M. T. Vilhena ${ }^{\text {a }}$ \\ ${ }^{a}$ Universidade Federal do Rio Grande do Sul, Grupo de Estudos Nucleares, 90050-170, Porto Alegre, RS, Brazil \\ ${ }^{b}$ Comissão Nacional de Energia Nuclear, 22290-901, Rio de Janeiro, RJ, Brazil
}

luizfelipe.fcb@gmail.com

\begin{abstract}
In this work the latest developments a Monte Carlo simulator with continuous energy is reported. This simulator makes use of a sum of three probability distributions to represent the neutron spectrum. Two distributions have known shape, but have varying population of neutrons in time, and these are the fission neutron spectrum and the Maxwell-Boltzmann distribution. The third distribution has an a priori unknown and possibly variable shape with time and is determined from parametrizations of Monte Carlo simulation. In this work the possible neutron-matter interactions are simulated with exception of the up-scattering of neutrons. In order to preserve the thermal spectrum, neutrons are selected stochastically as being part of the thermal population and have an energy attributed to them taken from a Maxwellian distribution, such an approximation is valid due to the fact that for fast neutrons up-scattering occurrence is irrelevant, being only appreciable at low energies. It is then shown how this procedure can emulate the up-scattering effect by the increase in the kinetic energy of the neutron population. Since the simulator uses tags to identify the reactions it is possible not only to plot the distributions by neutron energy, but also by the type of interaction with matter and with the identification of the target nuclei involved in the process.
\end{abstract}

Keywords: Monte Carlo, neutron transport, continuous energy, reaction tagging, thermal neutrons identification.

\section{INTRODUCTION}


Neutron transport is relevant in a variety of applications as for instance industrial applications, such as structural examinations, in nuclear medicine with diagnostic and therapeutic procedures, or radiation protection and last not least nuclear energy production. Neutron propagation description is still a challenge, since there does not exist a continuous energy deposit formula as for instance ionizing particles. Moreover, between nuclear interactions, the neutron may be considered a free particle, which is also manifest in the small cross sections when compared to interaction that involve electromagnetic interactions. Hence, the present contribution is a part in a larger project, where in the long term we intend to provide an alternative program package for neutron transport and interactions for the GEANT platform [1], which, currently, utilizes the MORSE [2] code for neutron transport. In this context, the present work reports on the development of a Monte Carlo simulator for neutron propagation and associated nuclear reactions either for transient or stationary problems and considering genuine continuous energy dependence by cross section function calls. This feature was the novelty of the first step of the development [3], which was optimized and extended by new modules, more specifically the consequences of up-scattering were included. This extension allows to simulate thermal aspects of the nuclear reactions by neutrons. In the further, we line out the structure of the program and exemplify its use simulating a simplified scenario of a nuclear reactor core with full three dimensional space and time dependencies. Thus far, results are shown for a standalone version, with only the essential reactions implemented. Nevertheless, the tracking and interactions of neutrons, as well as the tagging capabilities, follow the GEANT's paradigm.

In the forthcoming discussion we present the state of developments of the simulator, which is based on a first approach documented in [3]. This program presents a novel procedure in which cross sections are obtained by continuous parametrizations in the range between $0 \mathrm{MeV}$ and $20 \mathrm{MeV}$, including resolved and unresolved resonances, and with a maximum deviation of the order of $\sim 1 \%$ from the data set ENDF [4]. Once, sectionally analytical parametrization are elaborated, the energy dependent cross section is calculated by a simple function call instead of accessing data bases by extrapolating between registered values. This procedure executes more efficiently than other models with respect to computational time that are found in the literature (GEANT [1], Serpent [5], MCBEND [6], MCNP [7], OpenMC [8], KENO [9], TRIPOLI [10]), where the cross sections are determined from interpolation of cross section from data bases. 
While the preliminary version [3] only considered down-scattering, an extension including also upscattering effects in the thermal region are implemented. The spectral neutron population balance in the thermal region is implemented by a statistical treatment with three partially intersecting distributions and their mutual connection by kinetics. A detailed discussion of the implementation and validation against an analytical model are shown by the authors in reference [11]. Two of these distributions are the fission distribution, for high neutron energies, and the Maxwell-Boltzmann distribution, for thermal neutron energies. Both distributions have fixed shape, but the population of neutrons in each of them typically changes with time. The third distribution is an intermediate one, which has, an a priori, unknown shape and population. The Maxwell-Boltzmann distribution is the one where up-scattering effects are considered, and all distributions are continuous over the whole range of energy present in the simulation.

\section{PROGRAM DESCRIPTION}

The program simulates each neutron history individually and thus allows for trivial parallelization. At the end of the simulation the whole ensemble of neutrons can be accessed in order to compute and visualize the data. The program runs the simulation for a given number of Monte Carlo steps, with a given initial neutron population. Both the Monte Carlo steps and the initial neutron population are tallied by the use of an optimized RAM usage. For the purposes of this work the initial population was of $10^{5}$ neutrons and the number of Monte Carlo steps was of 2500. All initial neutrons are members of the fission population.

It is noteworthy that all the seven parameters that are present in the Boltzmann transport equation are considered, where the path of free propagation is selected stochastically, and ends at a vertex with one of the implemented interactions. A comment is in order here, the tracking and the interaction scheme was optimized in the sense that each Monte Carlo step has an interaction, which increases computational efficiency, but at the cost of loosing a unique relation between Monte Carlo step and corresponding time interval. Thus, a Monte Carlo step may be related only to a time interval distribution, that may be constructed from the tallies. 
The program has, so far, only a handful of the most important cross sections implemented. These are presented in Table 1.

Table 1: Implemented nuclides and reactions.

\begin{tabular}{cc}
\hline Implemented nuclides & Implemented reactions \\
\hline${ }^{1} \mathrm{H}$ & Elastic scattering \\
${ }^{16} \mathrm{O}$ & Radiative capture \\
${ }^{235} U$ & Fission \\
${ }^{238} U$ &
\end{tabular}

To date delayed neutrons are not implemented yet. All neutrons emitted by fission have their energies sampled from the Watt distribution, given by Equation (1). Their direction of emittance is also isotropically distributed, and the number of neutrons emitted by fission is taken from a Bernoulli distribution between 2 and 3 with mean of 2.48 .

$$
\chi(E)=0.453 e^{-1.036 \mathrm{MeV}^{-1} E} \sinh \left(\sqrt{2.29 \mathrm{MeV}^{-1} E}\right)
$$

Differently from other neutron transport codes, the present one has one interaction per Monte Carlo Step that occurs at the end of the trajectory segment. The position of a reaction depends on the kinetic energy of the neutron, its position at the beginning of the step, the direction of movement and the total macroscopic cross sections respecting the chemical composition of the reactor core material along the trajectory. The final position of the track is determined by a stochastic selection for the length of the traveled path from an exponential distribution with mean equal to the mean free path. Once the new position is determined, a test if the neutron remains inside or escapes to the outside of the domain decides whether tallying continues or ends.

After the selection of the position of interaction the interaction itself is chosen. This procedure first selects the molecule of the interacting nuclide by the ratio of the total macroscopic cross section of the molecule to the total macroscopic cross section of the mixture. Subsequently the nuclide of interaction is chosen by the same procedure, this time considering the ratio of each nuclide total 
macroscopic cross section to the one of the molecule. Lastly the type of reaction is chosen by the ratio of each of the nuclide's possible reaction macroscopic cross sections to the nuclide's total macroscopic cross section.

In case of scattering, a new energy and a new direction in agreement with energy and momentum conservation is given to the neutron. A simplification of the program is that it considers the scattering as isotropic in the center of mass system. Strictly speaking, scattering is isotropic for low kinetic energies and small nuclei, however, as the collision energies become higher and/or target nuclei become larger, anisotropy increases. The scattering module of the simulator may be extended in order to take into account anisotropic scattering, simply by exchanging the angular probability distribution in the module, without affecting the structure or the other modules of the program.

The previous version of the simulator as reported in [3] considered down-scattering only. However, the closer neutrons approach thermal energies up-scattering becomes non negligible, due to the thermal motion of the target nuclei which is no longer vanishingly small in comparison to the neutron's kinetic energy.

Nevertheless, as soon as neutrons may be classified as thermal they are in equilibrium with the environment which allows to simplify the tracking and interaction procedure. Equilibrium implies conservation of the respective energy distribution, which from the microscopic point of view means that neutrons in the average gain as much kinetic energy in collisions as they loose, so that the only relevant stochastic quantity that shall be determined is the reaction rate.

The procedure to determine whether a neutron belongs thermal distribution relies on a random number between 0 and 1 which is compared to the Maxwell-Boltzmann cumulative distribution. For a number larger than the cumulative distribution the neutron is considered thermal. Since, in the thermal region the energy distribution in the average does not change, at an interaction these neutrons get assigned a new energy, sampled from the Maxwell-Boltzmann probability distribution. According to [12], for large well-moderated reactors with homogeneous temperature, the MaxwellBoltzmann distribution can be a good approximation for the thermal neutron spectrum. This, however, is not the case for heterogeneous reactors with large temperature gradients, in which a more detailed treatment is required.

In the present work, which is to show how the simulator works at its present state, a rather simplified reactor core geometry was considered. More specifically, a cube with edges of 
dimensions $400 \mathrm{~cm} \times 400 \mathrm{~cm} \times 400 \mathrm{~cm}$, which contains a homogeneous mixture of water and uranium dioxide, enriched to $0.73 \%$ and the latter occupies $25 \%$ of the volume.

\section{RESULTS}

The total neutron population and the population of each of the aforementioned distributions per Monte Carlo step is presented Figure 1. It is possible to perceive that, excluding a few initial steps in which the bias of starting the simulation with purely fission neutrons, the reactor assumes a recurrent regime near a critical state.

Figure 1: Neutron population.

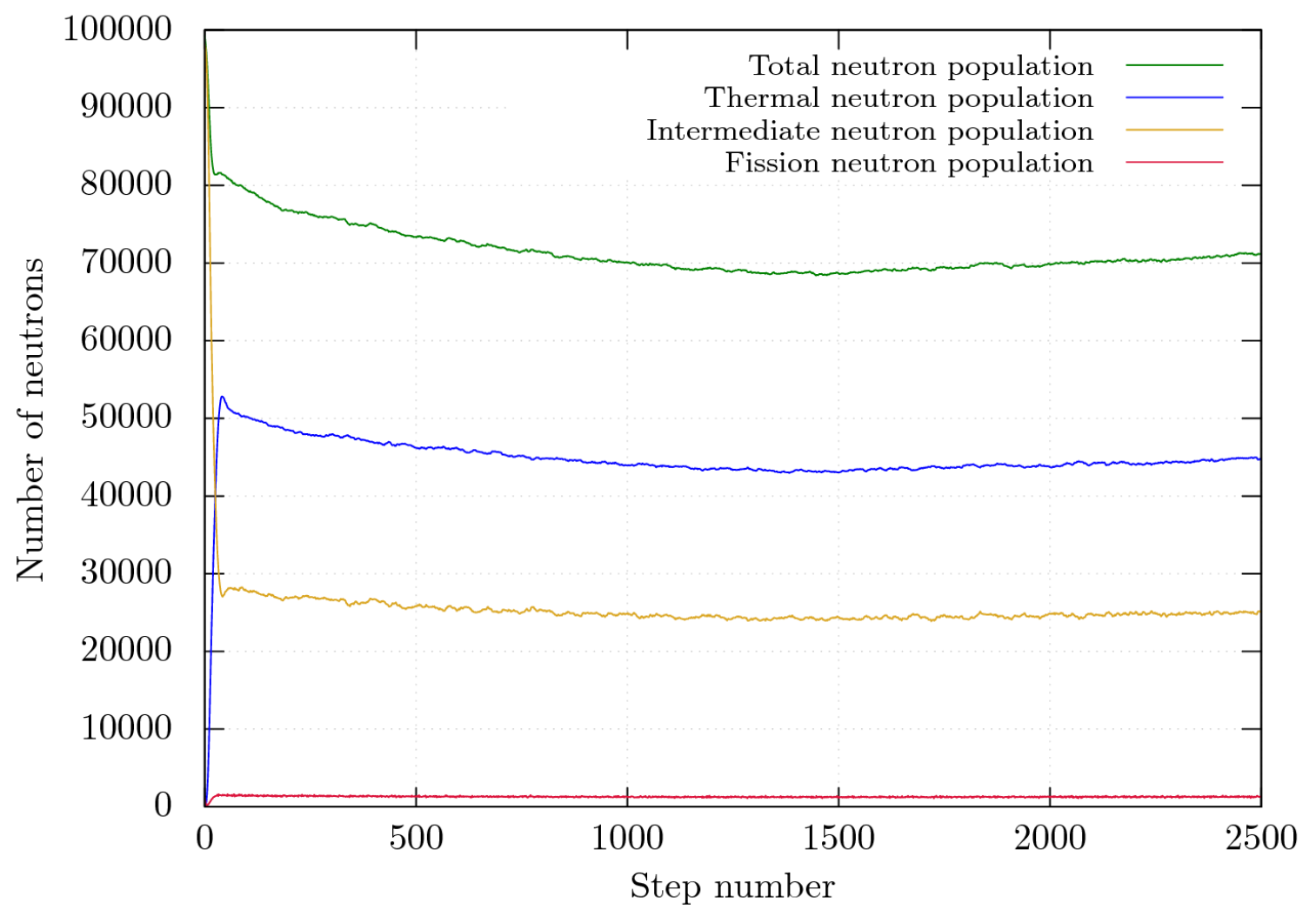

It is noteworthy that the ratio between the population of each distribution is almost constant. As can be seen in Figure 2 the ratio of each population to the total has small fluctuations around a mean value, again neglecting the bias of the initial steps. 
Figure 2: Population ratio.

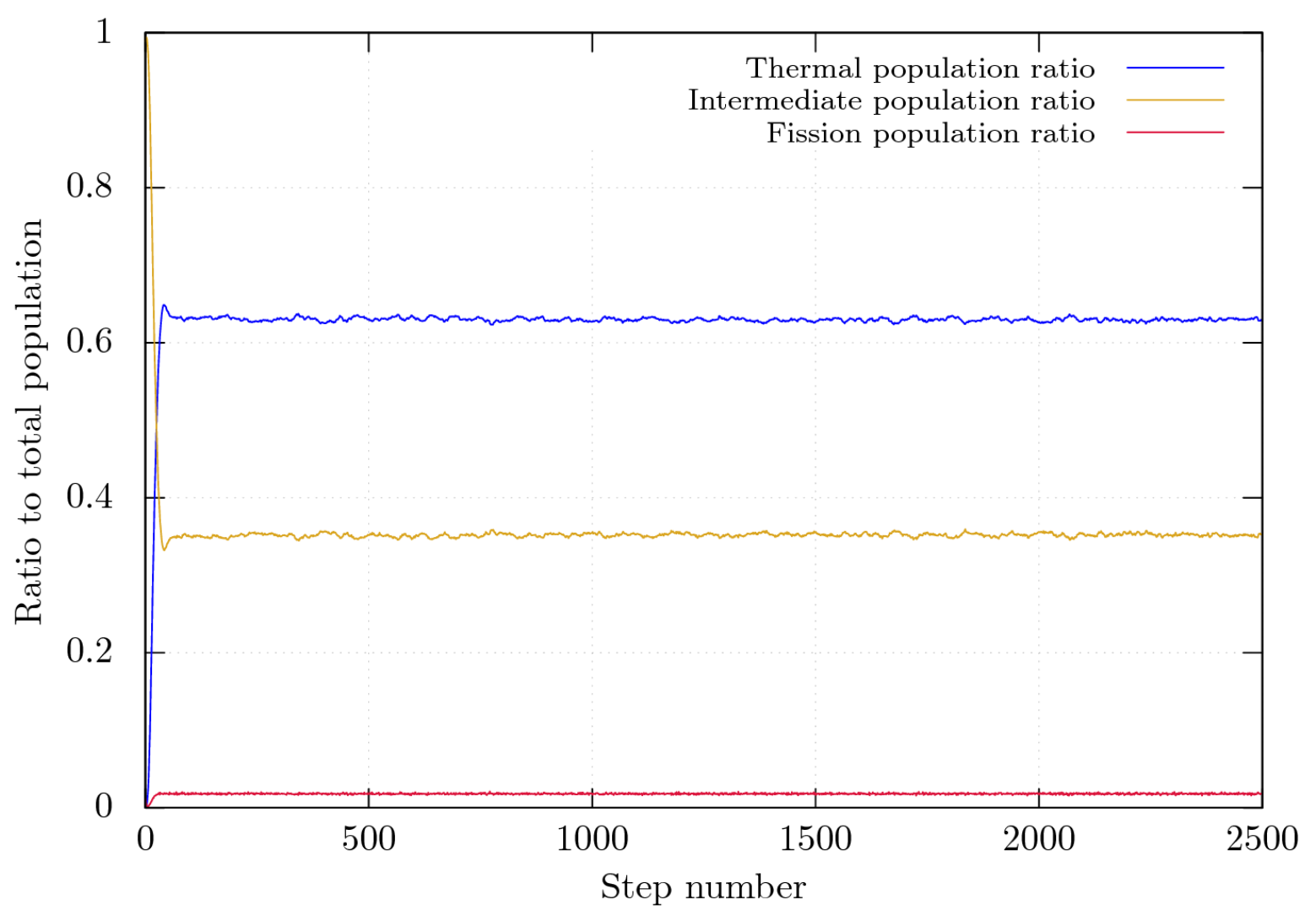

Another important result is the effective multiplication factor, this is presented in Figure 3. It is possible to perceive that this factor fluctuates around unity during the greater part of the simulation and then decreases. This behavior is explained by the program termination and the fact that no neutron can surpass step number 2500 .

It is also possible to portray the neutron spectrum, not only for the whole of the population, but for each individual distribution, this is done in Figure 4. In order to obtain these spectra, all neutron energies after steps number 100 were considered. The first 100 steps were neglected in order to consider only the recurrent regime. Note, that the aforementioned simulator allows the intersection of distributions and a given neutron energy is not bound to any distribution.

Figure 3: Effective multiplication factor. 


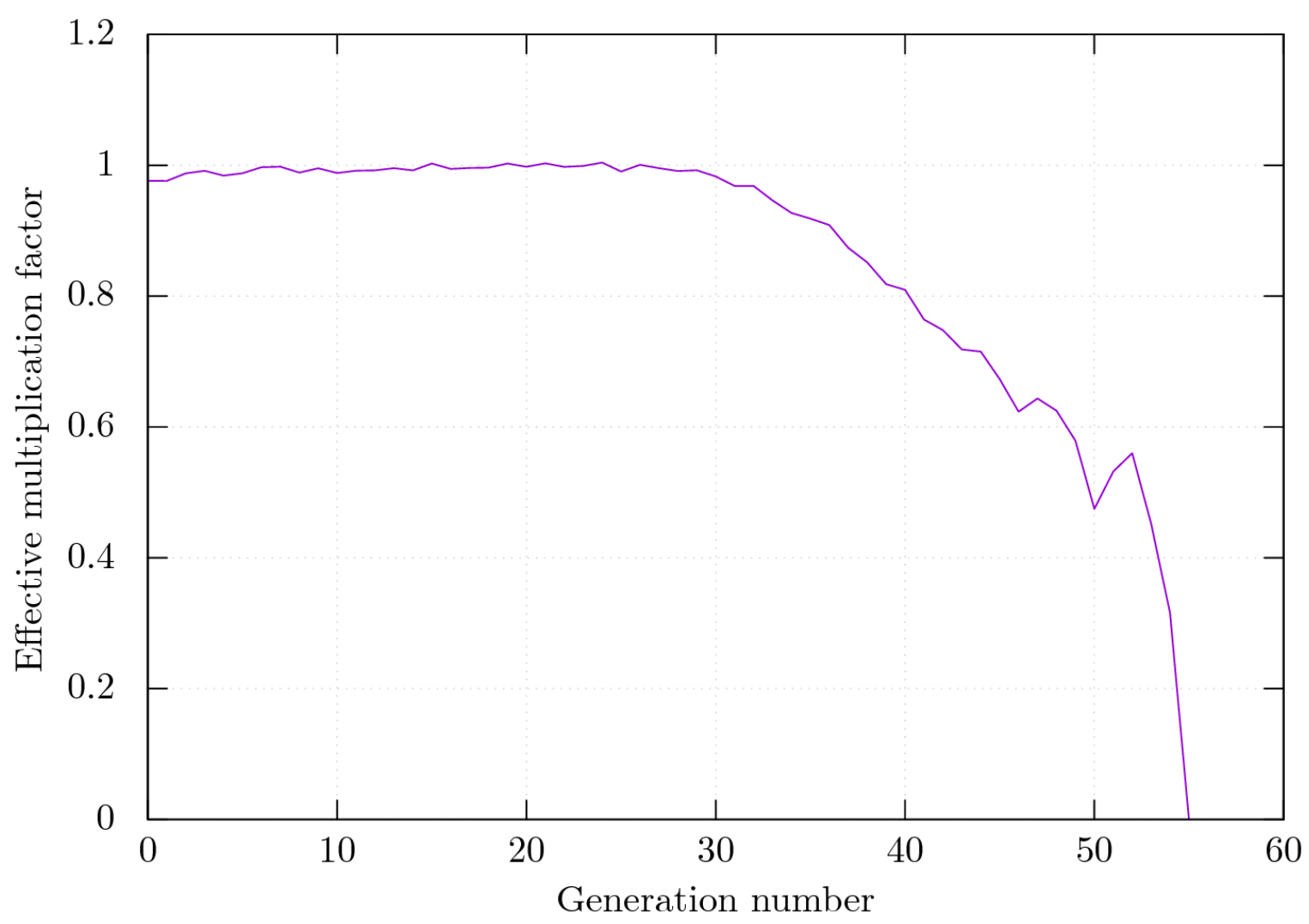

\section{CONCLUSIONS}

In this work we showed the state of developments of a new Monte Carlo transport code for neutron interactions. Firstly the simulator was successful in achieving a recurrent regime, for which the number of neutrons and the effective multiplication factor were shown. Also, the simulator correctly identified the population to which each neutron belonged, which, after the initial bias was gone, did not change any further, with the exemption of statistical fluctuations.

We also showed the neutron spectrum for all three distributions as well as for the total population. The importance of this result comes from the fact that both Watt and Maxwell-Boltzmann distributions have known shape, although their normalization can vary, but the remaining part of the spectrum is composed by a distribution of a priori unknown shape, which depends on details of the reactor geometry and chemical components. This third distribution, for neutrons that have intermediate ener- 
Figure 4: Neutron spectrum.

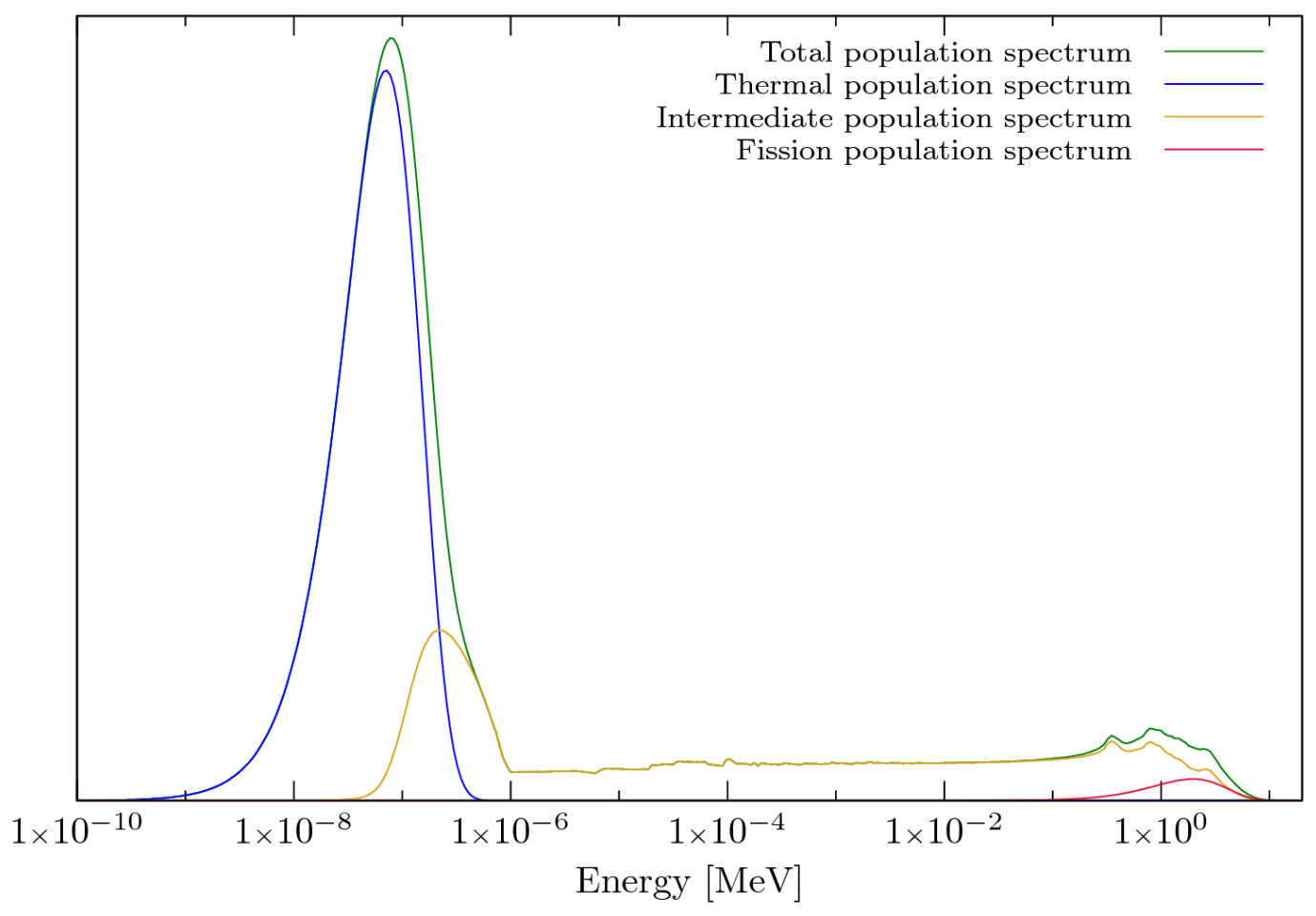

Figure 5: Neutron thermalisation.

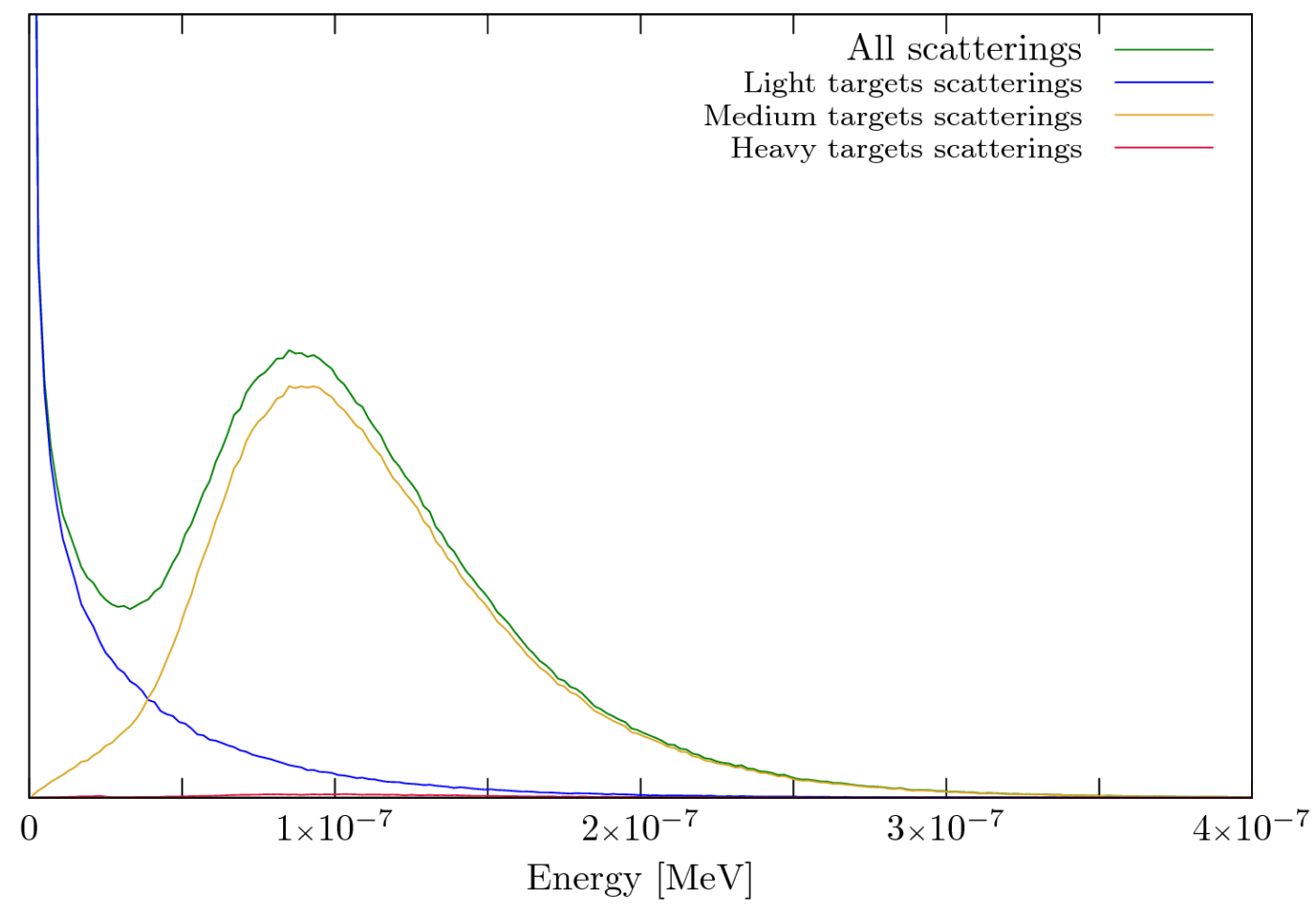


gies between fission and thermal energies, was successfully identified both in shape and in normalization.

Lastly, in Figure 5 we showed the neutrons as they were selected as part of the thermal population. This results also differentiates the contribution of the scattering targets to the thermalisation of neutrons. From this result it is possible to asses not only the error of considering the scattering target at rest, but also the correction done by the sampling of the neutron energy from a Maxwellian distribution.

In future works we intend to implement the program as a package for GEANT as an alternative for the current MORSE code for neutron transport and update the scattering module by anisotropic and energy dependent angular distributions. Further, also Doppler broadening due to temperature effects will be implemented in the next version of the simulator. 


\section{ACKNOWLEDGMENTS}

The authors thank CNPq and CAPES for financial support.

\section{REFERENCES}

[1] AgOSTINElli, S., Allison, J., AMAKO, K., APOSTOLAKIS, J., ARAUJO, H., ARCE, P., ASAI, M., AXEN, D., BANERJEE, S., BARRAND, G., AND OTHERS Geant4 - a simulation toolkit. Nuclear instruments and methods in physics research section A: Accelerators, Spectrometers, Detectors and Associated Equipment, v. 506, n. 3, p. 250-303, 2003.

[2] EMMETT, M.B. AND OTHERS The MORSE Monte Carlo radiation transport code system, Oak Ridge National Laboratory, USA, 1975

[3] DE CAMARGO, D.Q., BODMANN, B.E.J., VILHENA, M.T., BOGADO LEITE, S.Q., ALVIM, A.C.M. A stochastic model for neutrons simulation considering the spectrum and nuclear properties with continuous dependence of energy. Progress in Nuclear Energy, v. 69, p. 59-63. 2013.

[4] CHADWICK, M.B., HERMAN, M., OBLOŽINSKỲ, P., DUNN, M., DANON, Y., KAHLER, A.C., SMITH, D., PRITYCHENKO, B., ARBANAS, G., ARCILlA, R. AND OTHERS ENDF/B-VII.1 nuclear data for science and technology: cross sections, covariances, fission product yields and decay data. Nuclear Data Sheets, v. 112, n. 12, p. 2887-2996, 2011.

[5] LEPPÄNEN, J. Serpent - a continuous-energy Monte Carlo reactor physics burnup calculation code. VTT Technical Research Centre of Finland, 2013.

[6] COWAN, P., DOBSON, G., MARTIN, J. Release of MCBEND 11. Proc. 12th International Conference on Radiation Shielding (ICRS-12) and 17th Topical Meeting on Radiation Protection and Shielding (RPSD-2012), 2013.

[7] BRIESMEISTER, J. AND OTHERS, MCNPTM-A general Monte Carlo N-particle transport code. LA-13709-M, Los Alamos National Laboratory, v. 4C, 2000.

[8] ROMANO, P.K., FORGET, B. The OpenMC Monte Carlo particle transport code. Annals of Nuclear Energy, v. 51, p. 274-281, 2013. 
[9] PETRIE, L.M., CROSS, N.F. KENO IV: An improved Monte Carlo criticality program. Oak Ridge National Laboratory, USA, 1975.

[10] BOTH, J.P., MAZZOLO, A., PENELIAU, Y., PETIT, O., ROESSLINGER, B. User manual for version 4.3 of the Tripoli-4 Monte-Carlo method particle transport computer code., 2003.

[11] BARCELLOS, L.F.F. CHAVES, BODMANN, B.E.J., LEITE, S.Q. BOGADO, VILHENA, M.T. On a continuous energy Monte Carlo simulator for neutron transport: Optimisation with fission, intermediate and thermal distributions. Integral Methods in Science and Engineering, Volume 2, Constanda, C. and others (eds.), Birkhäuser, Cham, 2017. p. 1-10.

[12] BELL, G. AND GLASSTONE, S. Nuclear Reactor Theory. Division of Technical Information, US Atomic Energy Commission, 1970. 